Computing and Informatics, Vol. 40, 2021, 408-427, doi: 10.31577/cai_2021_2_408

\title{
OPTIMAL ALLOCATION OF CHARGING STATIONS FOR ELECTRIC VEHICLES USING PROBABILISTIC ROUTE SELECTION
}

\author{
Henrik Fredriksson, Mattias DAHL \\ Department of Mathematics and Natural Sciences \\ Blekinge Institute of Technology \\ 37179 Karlskrona, Sweden \\ e-mail: $\{$ henrik.fredriksson, mattias.dahl\}@bth.se
}

\author{
Johan Holmgren \\ Department of Computer Science and Media Technology \\ Malmö University \\ 20506 Malmö, Sweden \\ e-mail: johan.holmgren@mau.se
}

\begin{abstract}
Electric vehicles (EVs) are environmentally friendly and are considered to be a promising approach toward a green transportation infrastructure with lower greenhouse gas emissions. However, the limited driving range of EVs demands a strategic allocation of charging facilities, hence providing recharging opportunities that help reduce EV owners' anxiety about their vehicles' range. In this paper, we study a set covering method where self-avoiding walks are utilized to find the most significant locations for charging stations. In the corresponding optimization problem, we derive a lower bound of the number of charging stations in a transportation network to obtain full coverage of the most probable routes. The proposed method is applied to a transportation network of the southern part of Sweden.
\end{abstract}

Keywords: Charging stations, electric vehicle, transportation network, optimal placement, self-avoiding random walk

Mathematics Subject Classification 2010: 90-C06, 90-B20, 90-B80 


\section{INTRODUCTION}

The sale rate of electric vehicles (EVs) has been growing rapidly over the past ten years, and there is a need to adapt the current transportation infrastructure to meet future recharging demands. Increased use of EVs - including both plug-in hybrid electric vehicles (PHEVs) and battery electric vehicles (BEVs) - has been recognized as a promising, sustainable approach to lowering traffic emissions, including greenhouse gases [1]. However, their limited driving range and the scarcity of public accessible charging stations prevent EVs from gaining widespread market acceptance [2]. Psychological stress caused by the fear that the vehicle will run out of energy and be stranded is referred to as range anxiety [3]. As a consequence of range anxiety, EV owners may use their electric-powered vehicles for short trips exclusively, with the result that they require an additional vehicle for longer trips. In a small survey with 58 participants from 2011, Skippon and Garwood [4] report that consumers might consider an EV as their main car or second car if it had a range of 150 miles $(241 \mathrm{~km})$ or 100 miles $(161 \mathrm{~km})$, respectively. In a more recent survey, from 2016, Skippon et al. [5] report that consumers' desired driving ranges for EVs have substantially increased. The results show that people who have driven a modern EV would consider having an EV as the main car if the driving range is 200 miles $(322 \mathrm{~km})$ and as the second car if the driving range is 150 miles. Also, the study by Jensen et al. [6] confirms that the limited driving range is a concern for the acceptance of EVs. The study reports that the EVs' driving ranges do not match the expectations of consumers, after they use EVs for a trial period. Thus, as decision-makers and infrastructure planners consider to gain market acceptance for EVs, it will be important to determine how to best allocate charging stations to compensate for the current limited capacity of EV batteries. A strategic allocation of accessible charging facilities or battery swap stations may reduce the range anxiety of EV owners. Hence, an important step in addressing the problem of allocating of the charging station is to identify the routes in the transportation network that are most likely to be used to serve as many EV drivers as possible. Additionally, a desirable outcome of a charging station allocation is that every vehicle that drive around in the network, regardless of its position, should be able to reach a charging station before it runs out of energy. A strategic deployment of charging infrastructure may also minimize the initial cost of the installment of new charging facilities and relieve the load on the electrical power system [7].

In the current paper, we propose a novel solution procedure for the set covering problem for the allocation of EV charging stations. The basic formulation of the set covering problem is to minimize the number of charging stations such that each route is covered by at least one charging station [8, 9]. The set covering formulation, among other methods of deploying a public charging infrastructure, has received substantial attention from the research community. In what follows, we give a brief review of some of the methods that have been proposed in the literature. In Wang and Lin [10], a flow-based set covering method is proposed to minimize the cost of 
installation of charging facilities such as fast-refueling stations and battery exchange stations. The optimization method for the flow-based set covering method is based on the following vehicle-routing logic: the greater the distance a vehicle is driving, the more likely it is that the vehicle will require refueling. An extension to the problem is to consider a dual objective model to minimize the installation cost and maximize the population coverage, by combining the flow-based set covering model and the traditional set covering model [11. Wang [11] considers the allocation of charging stations for electric scooters, where the aim is to minimize the total installation cost. The model by Wang [1] is extended by Wang and Lin [12 to consider facility budget constraints, multiple types of recharging stations, and vehicle routing behavior. The types of charging stations include slow- and fast-recharging stations as well as battery swap stations. The case study presented in [12] shows that the results achieved with mixed-type charging facilities are better than those achieved with single-type facilities. The refueling logic requires numerous binary variables, which makes the problem hard to solve. When a flexible expanded network method is used, as proposed by MirHassani and Ebrazi [13], the solution time of the flow-based set covering method is significantly reduced. Wen et al. 14 consider both the problem of how to maximize the flow coverage with a fixed number of available charging stations and the problem of how to minimize the number of charging stations to obtain full coverage. In both models, the limited driving range of EVs is addressed by partitioning routes into sub-routes according to recharging logic. In the above-mentioned model, the general assumption is that vehicles would only consider routes that are the shortest or have the least traveling time between origin and destination. Li and Huang [15], Huang and Zhou [16], and Hosseini et al. [17] use the concept of deviation path. In their models, the shortest-path assumption is relaxed by the assumption that EV users are willing to slightly deviate from their preferred trips to ensure that they can refuel en route to their destinations. Frade et al. [18] present a mixed-integer optimization problem to maximize the coverage of both daytime and nighttime demand within an acceptable level of service for a neighbourhood in Lisbon. Funke et al. [19] propose a framework based on the hitting set problem, which aims to guarantee energy supply for all shortest paths in the network.

Another method commonly used to locate charging facilities and battery swap stations is based in flow capturing models [20, 21]. The Flow Refueling Location Model (FRLM) aims to locate a fixed number of charging stations in the nodes in such a way that the total number of vehicles can be refueled within their limited driving range [22]. Further, if it is possible to locate charging stations both along links and in nodes, the coverage of the network may be substantially improved compared to when nodes are the only candidate sites [23. A more realistic extension to the FRLM is to consider the charging capacity of the allocated facilities [24]. In the capacitated FRLM, the location variables are not binary but non-negative integers, meaning that multiple charging facilities could be located to serve as many vehicles as possible. Based on a flow-capturing model, Lim and Kuby [25] developed three heuristic algorithms for locating alternative-fuel stations. Solving the 
FRLM is usually a two-stage process; the first stage generates combinations of candidate locations, and the second stage uses these combinations to locate charging stations to maximize the number of refueled vehicles. Capar and Kuby [26] developed a method to solve the FRLM in one stage. To plan and design an infrastructure complete with battery swapping stations and battery management, Mak et al. 27] study the robust location problem of battery swapping stations under demand uncertainty.

If available, Global Position System (GPS) data can be utilized to support the allocation of charging stations. GPS data can, for example, be collected from taxis to obtain vehicle travel patterns, which are used to allocate charging stations [28, 29, 30, 31]. GPS travel survey data can also be used to simulate vehicles' driving and charging behavior to optimally locate charging stations such that the number of missed trips is minimized 32. Additional data, such as initial battery level of the vehicle and charging mode (normal or fast), together with GPS data, may provide useful insights on the charging behavior of EVs [33]. However, the applicability of methodologies based on GPS data is limited, due to the lack of data available for research purposes 34 .

To capture the interaction between the availability of charging stations and the route choices of drivers, several studies use traffic assignment models to identify the locations of charging stations. Traffic assignment models are multi-commodity flow problems under some given optimal or equilibrium routing principle. He et al. 35 propose a bi-level traffic assignment model. The upper level allocates a fixed number of charging stations such that the number of vehicles that use a charging station is maximized, while user equilibrium of route choice together with the EV's limited range is considered in the lower level. He et al. 36 propose a framework to capture the interactions between the locations of charging stations, electricity prices, route choices, and recharging time, which is solved by an active-set algorithm. Additionally, He et al. [37] present three different network equilibrium models, where the different flow dependencies and energy consumption are integrated. A similar model considers drivers' spontaneous adjustments and the interactions of travel and recharging decisions [38].

Despite the extensive work in academia, the process of allocating charging stations is still a challenging problem in real-world scenarios due to legal, physical, and financial constraints [34. Typically, constraints are much stricter and more complex than is assumed in studies, and there is a need for data and knowledge to fully understand the impacts of charging infrastructure concerning location, installation, operation, and future maintenance.

The current paper extends the paper by Fredriksson et al. [39] where an iterative solution procedure to optimally allocate charging stations for EVs is proposed. In particular, the contribution of the current paper is an explicit termination criteria for the iterative method, along with an improved route identification method to capture driving behavior. The studied problem is formulated as a set covering problem where the constraints in the associated integer problem are obtained by self-avoiding random walks. In the random walks, a probabilistic rule determined by link flows 
is applied in each node to select the next node in the walk. By iteratively adding constraints and solving sub-problems, we obtain a lower bound approximation of the minimal number of charging stations required to cover a transportation network without route enumeration.

The paper is organized as follows: In Section 2 we describe the model and the problem formulation. The proposed solution algorithm is explained in Section 3. Numerical results are presented in Section 4, and Section 5 concludes this paper and discusses some future research directions.

\section{PROBLEM FORMULATION}

A transportation network is described by a set of nodes $N=\{1,2, \ldots, n\}$ and a set of routes $R$. For each route $r \in R$, let $\delta_{i r}=1$ if a vehicle is visiting node $i \in N$ while traveling on route $r$, and $\delta_{i r}=0$ otherwise. Let $x_{i}$ be a binary variable where $x_{i}=1$ if a charging station is allocated in the node $i \in N$, and otherwise $x_{i}=0$. An allocation of charging stations is mathematically defined by a vector $\boldsymbol{x}=\left(x_{1}, x_{2}, \ldots, x_{n}\right) \in\{0,1\}^{n}$. The driving range is the maximal distance a vehicle can drive without recharging and is denoted by $d_{\max }$. Let $d_{w}^{E}$ be the Euclidean distance from the start node to the end node in a self-avoiding walk $w$. A self-avoiding walk $w$ is a route if $d_{w}^{E}>d_{\min }^{E}$ where $d_{\min }^{E}$ is a positive real number.

The problem studied in this paper is formulated as a set covering problem and is based on covered known routes. A route $r \in R$ is covered if at least one charging station is placed in one of its nodes. The route cover criteria for a route $r$ correspond to the inequality

$$
\sum_{i \in N} \delta_{i r} x_{i} \geq 1
$$

The set covering problem for the allocation of charging stations can be described as follows: Given a transportation network, find the set of all routes $R$ and the minimal number of charging stations and their locations such that each route $r \in R$ is covered. The optimization part of the problem corresponds to the optimization program

$$
\text { (P) } z=\min _{\boldsymbol{x} \in\{0,1\}^{n}}\left\{\sum_{i \in N} x_{i}: \sum_{i \in N} \delta_{i r} x_{i} \geq 1, \forall r \in R\right\} .
$$

Theoretically, even for a rather small transportation network, a complete route enumeration can lead to an unmanageable number of constraints in our set covering problem. Furthermore, if a complete route enumeration is available, we strongly believe that many of these routes may not be used at all by any vehicle. Instead of trying to find the set of all routes, we claim that it is sufficient to identify the most probable sub-routes (sub-routes that are most likely to be used), which, however, is far from a trivial task in large transportation networks. For simplicity, we refer to these sub-routes as routes. We assume that all routes $r \in R$ have a maximum 
length $d_{\text {max }}$. Hence, vehicles driving distances shorter or longer than this maximum distance cover fractions of routes or multiple routes, respectively. We describe our route identification method in Section 3.2, where we also discuss how we deal with the limited driving range of EVs.

\section{OPTIMAL PLACEMENT OF CHARGING STATIONS}

In this section we describe the solution procedure for our set covering problem. We also discuss how we obtain the routes that will be iteratively added to our model.

\subsection{Solution Procedure}

A solution to the charging station allocation problem is obtained by iteratively solving sub-problems $\left(P^{(k)}\right)$ of our problem $(P)$, where the sub-problems are based on subsets of routes $R_{k} \subset R$. The sub-problems yield a sequence of optimal solutions with a monotonically increasing minimum number of charging stations, converging to the optimum value of problem $(P)$. Our aim is to search and select routes in the network to achieve a solution with coverage of the most probable routes. To this end, we exploit probabilistic self-avoiding random walks to identify routes in the transportation network. The identified routes are iteratively generated and added as constraints to the integer problem under consideration. The approximation technique proposed to solve our problem is based on integer programming, and the outline of the method can be described with the following basic steps:

0 . Initialization: Set iteration counter $k=0$, and $R_{k}=\emptyset$. Fix $x_{i}=1$ if a charging station is already allocated in node $i \in N$.

1. Given a reference set $R_{k} \subset R$, solve the sub-problem

$$
\left(P^{(k)}\right) \quad z^{(k)}=\min _{\boldsymbol{x} \in\{0,1\}^{n}}\left\{\sum_{i \in N} x_{i}: \sum_{i \in N} \delta_{i r} x_{i} \geq 1, \forall r \in R_{k}\right\}
$$

yielding the solution vector $\boldsymbol{x}^{(k)}$.

2. Define the entering index by a route $r_{e} \in R$ satisfying $\sum_{i \in N} \delta_{i r_{e}} x_{i}^{(k)}=0$. Stop with approximate optimal reference set $R_{k} \subset R$, if a new $r_{e}$ cannot be found according to some termination criteria.

3. Define the new reference set by $R_{k+1}=R_{k} \cup\left\{r_{e}\right\}$, set $k=k+1$, and go to step 1.

A flowchart of the solution procedure is depicted in Figure 1. At iteration $k$, the solution vector $\boldsymbol{x}^{(k)}$ is infeasible for the new reference set $R_{k+1}$ since the new entering index $r_{e}$ is uncovered. We can easily provide a feasible solution to subproblem $P^{(k+1)}$ by setting $x_{i}=1$ where $i=\min \left\{k: \delta_{k r_{e}}=1\right\}$. 
Without any prior knowledge about the routes in the network, it is computationally difficult to estimate the number of routes in a transportation network, and thereby to find a suitable, explicit termination criteria. For instance, the iterative method could be terminated when a sufficient number of constraints has been collected, when some search time for a new uncovered route is exceeded, or when a new uncovered route cannot be found within a certain number of iterations. In our numerical examples in Section 4 we apply the latter termination criteria. If a new uncovered route cannot be found after 10000 random walk attempts, we assume that the most commonly used routes in the network have been taken into consideration.

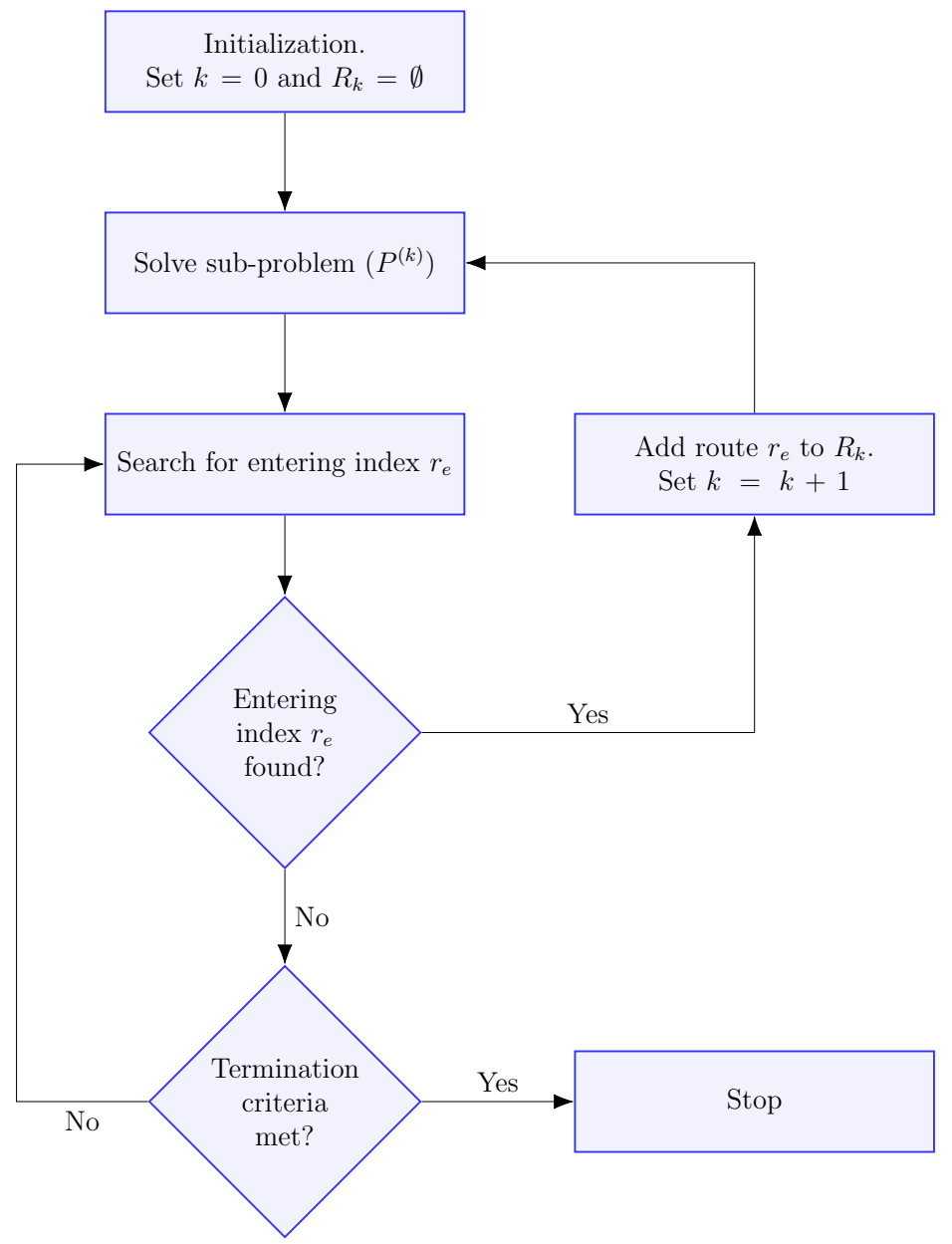

Figure 1. Flowchart for the solution procedure for our set covering problem 


\subsection{Self-Avoiding Random Walks}

As mentioned above, the constraint index $r_{e}$, which is chosen to enter the basis $R_{k}$ is defined by a route that does not satisfy the current solution, i.e., a constraint violation. By using the most probable routes in the network, we claim that when no entering index $r_{e}$ can be found according to some termination criteria, the most commonly used (probable) routes in the network will be covered by at least one charging station.

In practice, finding the complete route set $R$ is computationally difficult, and several models use a predefined set of routes. We use self-avoiding random walks as a search method to simulate the driving behavior of EV drivers to find new uncovered routes. A self-avoiding random walk is defined as a random walk with the restriction that it cannot revisit a node. Self-avoiding random walks can be more effective when exploring a transportation network and may model driving behavior more realistically than unrestricted random walks, since they cannot return to already visited nodes [40]. A probabilistic rule determined by collected link flows is applied at each node to select the next node among the neighbors of the current node, yielding a probabilistic self-avoiding random walk. Let $i \in N$ be the current node in the walk; then the probability of choosing the next neighboring node $j$ is

$$
p=\frac{\phi(i, j)}{\sum_{x \in N_{i}} \phi(i, x)} .
$$

Here, $N_{i} \subset N$ is the set of adjacent and unvisited nodes of the current node $i$, in the current walk under construction, and $\phi(i, j)$ is the flow on link $(i, j)$.

Link flows can be collected using temporary or permanent sensors, including pneumatic tubes, that are moved around in the network according to a periodic scheme. Obviously, the link flows do not provide as much information as other flows - e.g., origin-destination flows and route flows - but they are easier to obtain. Obviously, the link flows vary over time, but on an aggregate level, static link flows provide a sufficient approximation to generate the most probable routes in a transportation network. The collected link flows are utilized to model driving behavior in the network. We argue that this approach provides a reference for planning and charging infrastructure for the current transportation situation. Due to the current vehicle market, the collected link flow data are mainly constituted by vehicles with internal combustion engines fueled by gasoline or diesel. Although changes may occur depending on vehicle type, in this study we assume that travel patterns and driving behavior are the same for EVs and for gasoline or diesel-fueled vehicles.

Depending on the scenario under investigation, in our route generation process, we restrict our selection to only consider routes with maximum length $d_{\max }$, which is assumed to be the maximal distance a vehicle can to travel without passing a node equipped with a charging station. Thus, a partition of the full route into road segments, of appropriate lengths to handle the limited driving range of EVs is 
identified directly. Since the program optimally allocates charging stations such that each route is covered, a solution will ensure coverage of the most probable routes of length $d_{\text {max }}$. In other words, an EV cannot travel longer than $d_{\max }$ without passing a charging facility if it travels on the most probable route. In this way, we can adjust the parameter $d_{\max }$ to respect the driving range of an $\mathrm{EV}$.

Since we are demonstrating our proposed method on a government-controlled transportation network with roads between cities and metropolitan areas, we are especially interested in covering routes that are included in trips between communities. To avoid peculiar zigzag behaviors or U-turns, we only consider routes where the Euclidean distance is at least $d_{\min }^{E}$ between the start- and end-nodes of the route.

Each of the random walks begins in a randomly chosen node. If a walk reaches a node already equipped with a charging station, i.e., the route is already covered by the current solution, a new random walk is restarted in a randomly chosen node until a new unique entering index $r_{e}$, violating the constraints in the current subproblem, is found. The search method for the entering index $r_{e}$ is done by the following steps.

1. Start in a random node.

2. With probabilities according to Equation (4), randomly choose a neighbouring unvisited node (in the current walk).

(a) If the chosen node has a charging station allocated on it, discard the walk and go to Step 1.

(b) If the total length of the walk under construction exceeds $d_{\max }$, and the Euclidean distance between the first and current node exceeds $d_{\text {min }}^{E}$, add the walk to the problem as a constraint, solve the sub-problem, and go to Step 1.

(c) Otherwise, repeat Step 2.

Depending on the network under study, there is a margin of error concerning the limited driving range, since the generated walks are presumed to be slightly longer than $d_{\max }$. This error, however, can be alleviated by lowering $d_{\max }$.

As the number of constraints increases, it will be more and more difficult to find new uncovered routes, since vehicles are more likely to traverse a node already equipped with a charging station. Hence, our iterative process converges towards an optimal solution where the most probable routes are covered. Because the iterative solution procedure converges to an optimal solution with full coverage of the most probable routes in a transportation network, we argue that a solution using our proposed method is worthy of consideration since it does not contain routes that are less likely to be used.

\subsection{Outline of the Convergence}

The main idea of the optimization procedure is to iteratively solve sub-problems of our set covering problem and to continuously further extend the current sub- 
problem into a slightly larger problem. Since the problem is formulated as a set covering problem, a solution to the problem is not necessarily unique, but the iterative procedure is continuously striving for a solution with a minimal number of charging stations. The procedure ensures coverage and localizes common junctions of interest within the given transportation network. The main purpose of this procedure is to ensure that each charging station is placed in an environment that is of mutual interest to several of the found routes.

The behavior of convergence can be briefly outlined, and the emphasis is on an intuitive understanding for the optimization procedure. Assume that we are given the solution $\boldsymbol{x}^{k}$ to any sub-problem $P^{(k)}$. If we now add one single constraint where $\boldsymbol{x}^{k}$ violates the constraint, we can easily provide a feasible solution to iteration $k+1$ as described in Section 3.1. We now establish the following important inequality relations

$$
z^{(1)} \leq \ldots \leq z^{(k)} \leq z^{(k+1)} \leq \ldots \leq z .
$$

The first inequality is due to the fact that the optimal solution for problem $\left(P^{(k+1)}\right)$ is a feasible solution for problem $\left(P^{(k)}\right)$ in integer problem formulation (3) and provides an upper bound for $\left(P^{(k)}\right)$. The sequence $\left(z^{(k)}\right)$ is thus monotonically increasing and upper bounded. Convergence to the optimum value of $z^{(k)}$ is motivated by the last equality where the existence of a finite optimal reference set $R$ is established. We note, in this context, that the convergence of the algorithm is based on the assumption that numerical difficulties (such as randomness, size of network, etc.) are avoided due to the presence of a reliable software for the solution. In practice, the optimal value of $z$ will never be reached for largescale networks. However, the system yields an appropriate approximation, and therefore it is subject to continuous improvement with the aim of reaching the optimum.

In this presentation, we emphasize the simplicity and efficiency of this approach to finding a minimal number of charging stations and allocating them so that every route is covered. Since the procedure is based on a controlled selection of constraints, there are opportunities to add and fulfill requirements that make the procedure of selecting routes to model the transportation network even more realistic.

\section{COMPUTATIONAL STUDY}

To demonstrate our proposed method's effectiveness, we consider the network of the southernmost part of Sweden. This network is one of six traffic regions maintained by the Swedish Transport Administration. The network consists of 14500 nodes and 34500 links distributed over an area of approximately 44500 square kilometers, spread across five counties, and it is shown in Figure 2. The mean length of the links is $1.29 \mathrm{~km}$ with a standard deviation of $1.98 \mathrm{~km}$. The distribution of the link lengths in the network under study is shown in Figure 3 . Since the majority of the links are quite short, routes in our scenarios ranges $60 \mathrm{~km}$ to $100 \mathrm{~km}$ in length, and 
will consist of several nodes. Although, a random walk may have choose a node with low probability, according to the probabilistic rule (4), it will most likely reassemble with more probable links in a later stage. The link flows used in our study are based on real-world data collected from traffic counts in July 2018. The data was obtained from The Swedish National Road Database (NVDB) provided by the Swedish Transport Administration. In the optimization procedure, neighboring regions have not been taken into account.

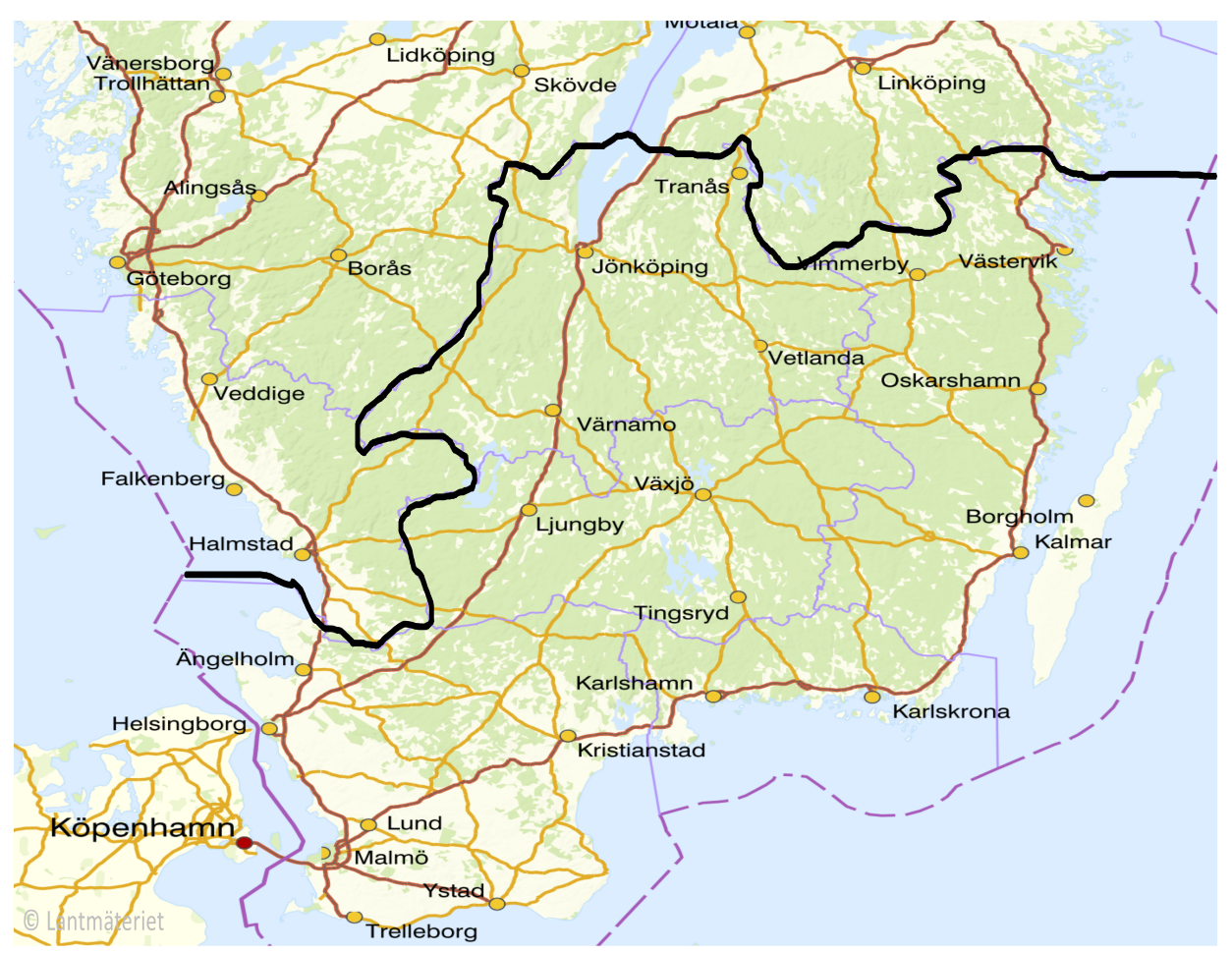

Figure 2. The national transportation main road map for the southern Sweden (area of interest is beneath the black border). The transportation network (all roads) consists of 14500 nodes 34500 links distributed over an area of 44500 square kilometers.

We implemented our problem-solving method to find allocations of charging stations in the numerical computing environment MATLAB ${ }^{\circledR}$ [4] and Gurobi Optimizer [42]. 


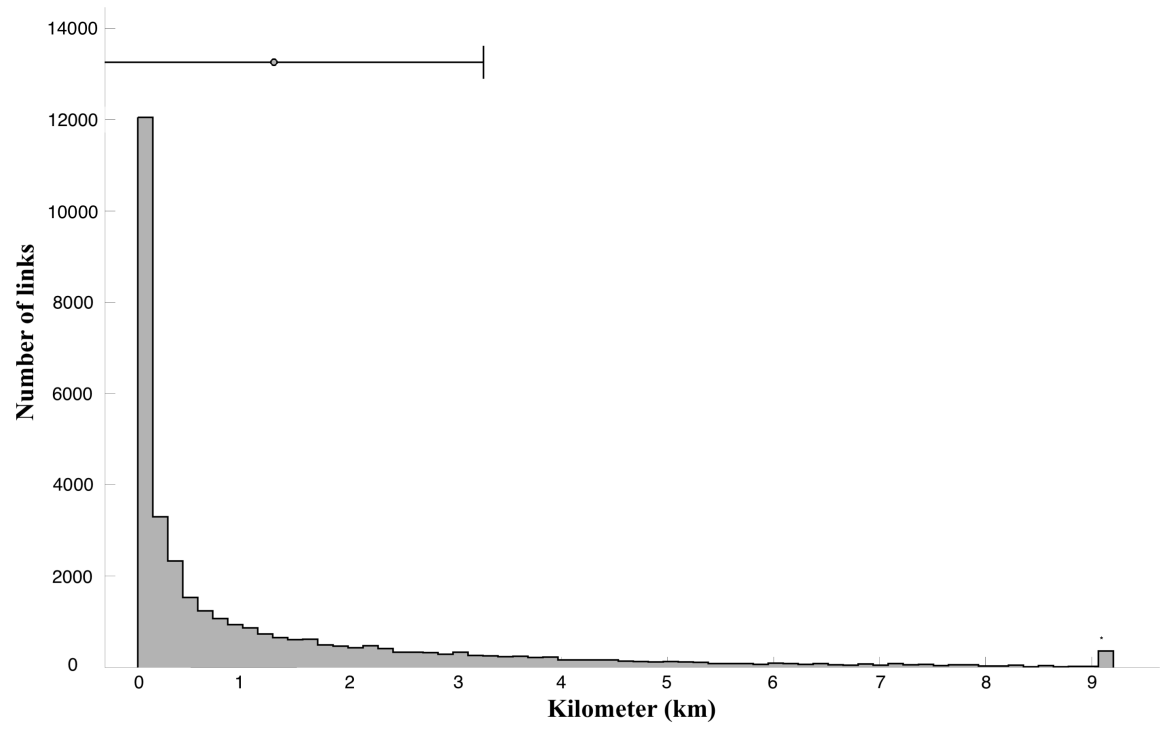

Figure 3. Distribution of link lengths in the network of southern Sweden, where the mean length of the links is $1.29 \mathrm{~km}$, with a standard deviation of $1.98 \mathrm{~km}$. The 339 points counted in the rightmost bin are links with length greater than $9.06 \mathrm{~km}$.

\subsection{Scenario Descriptions}

In our numerical evaluations we consider 3 different scenarios, each with varying maximal driving range $d_{\max }$, and minimal distance travelled $d_{\min }^{E}$. We study the number of charging stations required to obtain full coverage of all found routes. In all scenarios we used a maximum number of search iterations as termination criteria. If no new uncovered route $r_{e}$ could be found within 10000 iterations, the program was terminated. The number of constraints in the final iteration and the number of allocated charging stations for each scenario are shown in Table 11. As mentioned in Section 3.3, a solution to our set covering problem might not be unique, but it provides a lower bound of the number of charging stations required to cover each of a number of considered routes. Examples of solutions for each of the considered scenarios are illustrated in Figures 4, 5, and 6.

We emphasize that, since we use a network in the southernmost part of Sweden, the studied scenarios have a macroscopic approach. The allocation of charging stations has a focus outside urban and metropolitan areas, in this regard, the process of its optimization has a focus on the government-controlled transportation network.

As the numerical results show, the number of required charging stations decreases as $d_{\max }$ increases. For illustrative purposes, Figure 7 shows how the optimal placements of charging stations have evolved during the iterative process for Sce- 


\begin{tabular}{lrrrr}
\hline & $d_{\max }(\mathrm{km})$ & $d_{\min }^{E}(\mathrm{~km})$ & Constraints & Allocated Charging Stations \\
\hline Scenario 1 & 60 & 30 & 1177 & 203 \\
Scenario 2 & 80 & 40 & 1075 & 174 \\
Scenario 3 & 100 & 50 & 569 & 104 \\
\hline
\end{tabular}

Table 1. Number of constraints and number of allocated charging stations of the iterative set covering model for the three scenarios generated by different parameter settings of $d_{\max }$ and $d_{\min }^{E}$

nario 3 with $d_{\max }=100 \mathrm{~km}$ and $d_{\min }^{E}=50 \mathrm{~km}$. The figure shows all nodes that have been included in an optimal solution $\boldsymbol{x}^{(k)}$ for some iteration index $k$. As seen in the figure, numerous nodes have been considered as candidate sites for optimal placement to achieve coverage. The final allocation of charging stations for this scenario is depicted in Figure 6.

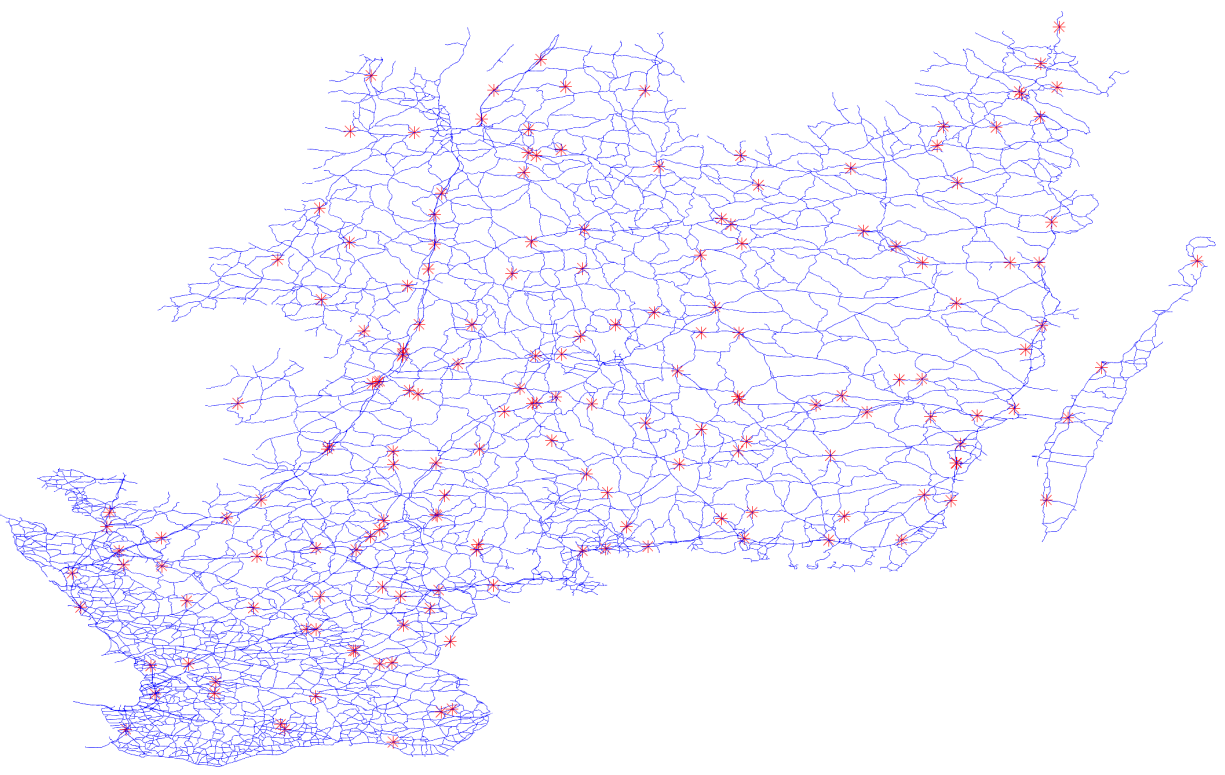

Figure 4. The allocation of charging stations for $d_{\max }=60 \mathrm{~km}$ and $d_{\min }^{E}=30 \mathrm{~km}$

\section{CONCLUSIONS AND FUTURE WORK}

EVs for both public and private transport seem to be a promising solution to reduce greenhouse gas emissions. However, the scarcity of available charging stations and the limited driving range of EVs, are two major barriers for the widespread adoption of EVs. In the current paper, we propose a node-based formulation of a set covering method to optimally allocate charging stations for EVs. The constraints in the 


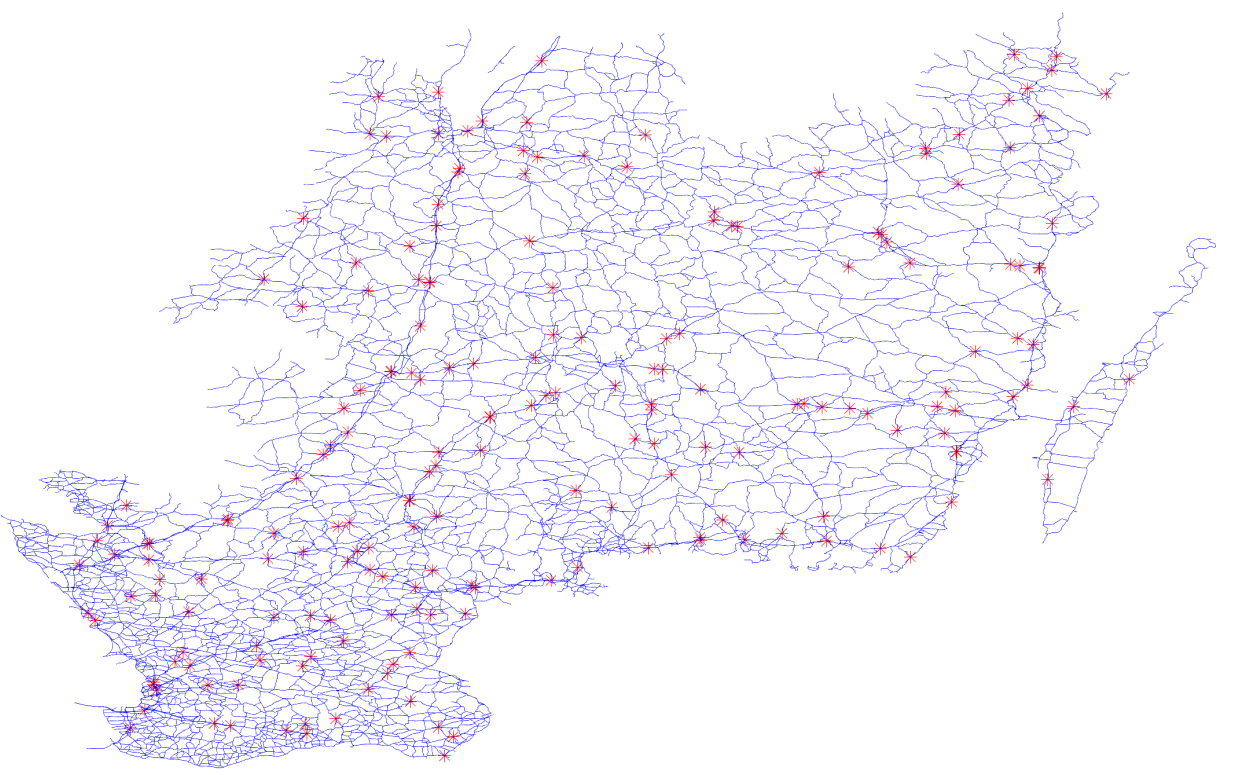

Figure 5. The allocation of charging stations for $d_{\max }=80 \mathrm{~km}$ and $d_{\min }^{E}=40 \mathrm{~km}$

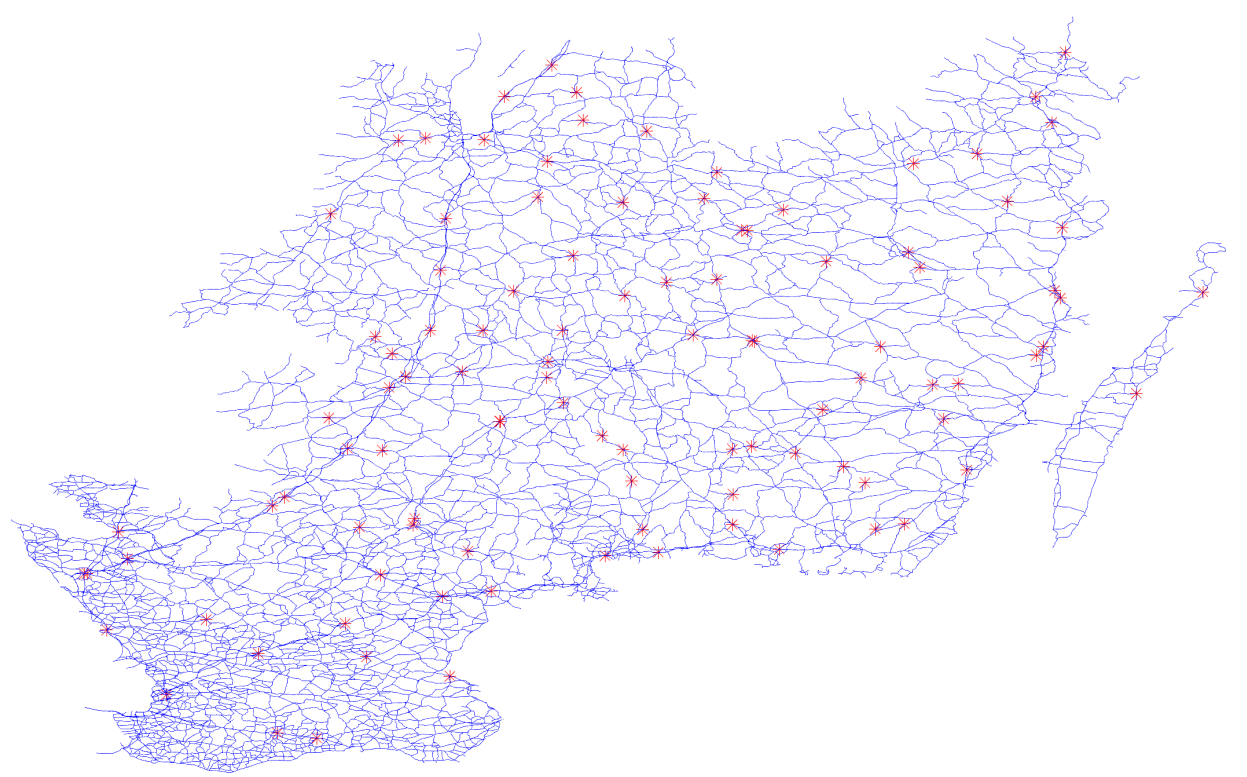

Figure 6 . The allocation of charging stations for $d_{\max }=100 \mathrm{~km}$ and $d_{\min }^{E}=50 \mathrm{~km}$ 


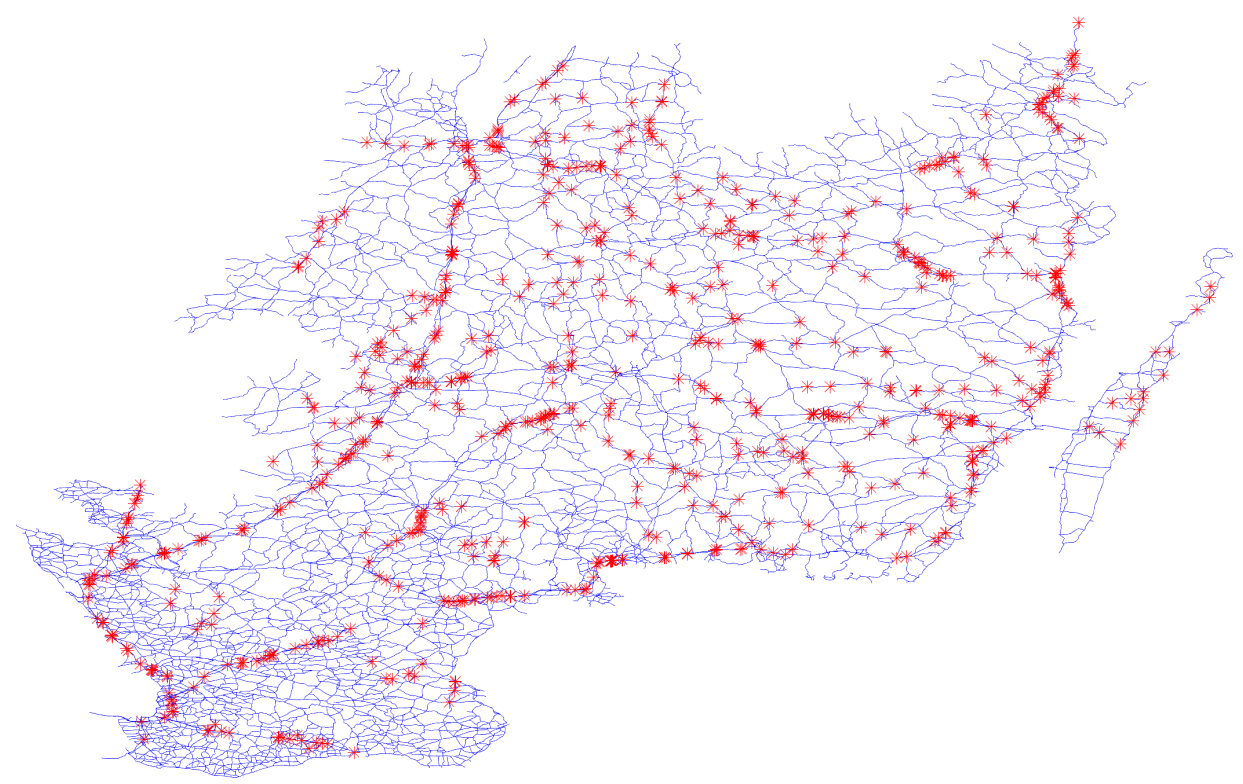

Figure 7. All candidate nodes for optimal allocation of charging stations for $d_{\max }=$ $100 \mathrm{~km}$ and $d_{\min }^{E}=50 \mathrm{~km}$

corresponding optimization problem are based on self-avoiding random walks along the links in the network and the problem is solved by a pruned integer program that can take the existing infrastructure into consideration. The iterative optimization procedure and the probabilistic route selection provide an approximation of the optimal allocation to obtain full coverage.

The computational results of the proposed iterative method, in the case study of the Southern Sweden transportation network, shows that the method is able to allocate charging stations optimally without numerical difficulties. The results indicate how charging facilities can be located strategically to cover the most probable routes for an EV fleet in the studied area, for the several different scenarios. In particular, the method provides a lower bound to obtain full coverage and localizes nodes that are of common interest for the routes in the transportation network. From the results of our computational study, in which we compared maximal driving distances of 60,80 , and 100 kilometers, respectively, we observe that the ability to extend the driving distance of EVs significantly reduces the need for EV charging stations. In particular, the number of allocated charging stations in our considered network dropped from 203 to 88 when $d_{\max }$ was increased from 60 to 100 kilometers. This means that the number of charging stations dropped by $56.7 \%$ when we increased $d_{\max }$ by $66.7 \%$. Hence, in addition to helping drivers to overcome the range anxiety, increasing the driving range of EVs also significantly reduces the need to build charging infrastructure. Further research based on 
the current work can be conducted in several directions. One direction could consider the inclusion of budgetary constraints and the recharging capacity of charging stations. Another research could be focused on the search method for uncovered routes. A third direction of further research includes the optimal allocation considering that charging will most likely occur in the beginning or the end of a trip.

\section{REFERENCES}

[1] Ohnishi, H.: Greenhouse Gas Reduction Strategies in the Transport Sector: Preliminary Report. Technical report, OECD/ITF Joint Transport Research Centre Working Group on GHG Reduction Strategies in the Transport Sector, OECD/ITF, Paris, 2008.

[2] Romm, J.: The Car and Fuel of the Future. Energy Policy, Vol. 34, 2006, No. 17, pp. 2609-2614, doi: 10.1016/j.enpol.2005.06.025.

[3] Neubauer, J.-Wood, E.: The Impact of Range Anxiety and Home, Workplace, and Public Charging Infrastructure on Simulated Battery Electric Vehicle Lifetime Utility. Journal of Power Sources, Vol. 257, 2014, pp. 12-20, doi: 10.1016/j.jpowsour.2014.01.075.

[4] Skippon, S.-Garwood, M.: Responses to Battery Electric Vehicles: UK Consumer Attitudes and Attributions of Symbolic Meaning Following Direct Experience to Reduce Psychological Distance. Transportation Research Part D: Transport and Environment, Vol. 16, 2011, No. 7, pp. 525-531, doi: 10.1016/j.trd.2011.05.005.

[5] Skippon, S. M.-Kinnear, N.-Lloyd, L.-Stannard, J.: How Experience of Use Influences Mass-Market Drivers' Willingness to Consider a Battery Electric Vehicle: A Randomised Controlled Trial. Transportation Research Part A: Policy and Practice, Vol. 92, 2016, pp. 26-42, doi: 10.1016/j.tra.2016.06.034.

[6] Fuendbo Jensen, A.-Cherchi, E.-Lindhard Mabit, S.: On the Stability of Preferences and Attitudes Before and After Experiencing an Electric Vehicle. Transportation Research Part D: Transport and Environment, Vol. 25, 2013, pp. 24-32, doi: $10.1016 /$ j.trd.2013.07.006.

[7] Hajimiragha, A.-Canizares, C. A.-Fowler, M. W.-Elkamel, A.: Optimal Transition to Plug-In Hybrid Electric Vehicles in Ontario, Canada, Considering the Electricity-Grid Limitations. IEEE Transactions on Industrial Electronics, Vol. 57, 2010, No. 2, pp. 690-701, doi: 10.1109/TIE.2009.2025711.

[8] Toregas, C.-Swain, R.-ReVelle, C.-Bergman, L.: The Location of Emergency Service Facilities. Operations Research, Vol. 19, 1971, No. 6, pp. 1363-1373, doi: $10.1287 /$ opre.19.6.1363.

[9] Daskin, M. S.: Network and Discrete Location: Models, Algorithms, and Applications. John Wiley \& Sons, Hoboken, New Jersey, 2013, doi: 10.1002/9781118537015.

[10] Wang, Y. W.-Lin, C. C.: Locating Road-Vehicle Refueling Stations. Transportation Research Part E: Logistics and Transportation Review, Vol. 45, 2009, No. 5, pp. 821-829, doi: $10.1016 /$ j.tre.2009.03.002 
[11] Wang, Y. W.: Locating Flow-Recharging Stations at Tourist Destinations to Serve Recreational Travelers. International Journal of Sustainable Transportation, Vol. 5, 2011, No. 3, pp. 153-171, doi: 10.1080/15568311003717199.

[12] Wang, Y.W.-Lin, C. C.: Locating Multiple Types of Recharging Stations for Battery-Powered Electric Vehicle Transport. Transportation Research Part E: Logistics and Transportation Review, Vol. 58, 2013, pp. 76-87, doi: 10.1016/j.tre.2013.07.003.

[13] MirHassani, S. A.-Ebrazi, R.: A Flexible Reformulation of the Refueling Station Location Problem. Transportation Science, Vol. 47, 2013, No. 4, pp. 617-628, doi: $10.1287 / \operatorname{trsc} .1120 .0430$

[14] Wen, M.-Laporte, G.-Madsen, O. B. G.-Nørrelund, A. V.-Olsen, A.: Locating Replenishment Stations for Electric Vehicles: Application to Danish Traffic Data. Journal of the Operational Research Society, Vol. 65, 2014, No. 10, pp. 1555-1561, doi: $10.1057 /$ jors.2013.100.

[15] Li, S.-HuAng, Y.: Heuristic Approaches for the Flow-Based Set Covering Problem with Deviation Paths. Transportation Research Part E: Logistics and Transportation Review, Vol. 72, 2014, pp. 144-158, doi: 10.1016/j.tre.2014.10.013.

[16] Huang, Y.-Zhou, Y.: An Optimization Framework for Workplace Charging Strategies. Transportation Research Part C: Emerging Technologies, Vol. 52, 2015, pp. 144-155, doi: 10.1016/j.trc.2015.01.022.

[17] Hosseini, M.-MirHassani, S. A.-Hooshmand, F.: Deviation-Flow Refueling Location Problem with Capacitated Facilities: Model and Algorithm. Transportation Research Part D: Transport and Environment, Vol. 54, 2017, pp. 269-281, doi: 10.1016/j.trd.2017.05.015.

[18] Frade, I.-Ribeiro, A.-Gonçalves, G.-Antunes, A. P.: Optimal Location of Charging Stations for Electric Vehicles in a Neighborhood in Lisbon, Portugal. Transportation Research Record, Vol. 2252, 2011, No. 1, pp. 91-98, doi: 10.3141/2252-12.

[19] Funke, S.-Nusser, A.-Storandt, S.: Placement of Loading Stations for Electric Vehicles: No Detours Necessary! Journal of Artificial Intelligence Research, Vol. 53, 2015, pp. 633-658, doi: 10.1613/jair.4688.

[20] Hodgson, M. J.: A Flow-Capturing Location-Allocation Model. Geographical Analysis, Vol. 22, 1990, No. 3, pp. 270-279, doi: 10.1111/j.1538-4632.1990.tb00210.x.

[21] Berman, O.-Larson, R. C.-Fouska, N.: Optimal Location of Discretionary Service Facilities. Transportation Science, Vol. 26, 1992, No. 3, pp. 201-211, doi: 10.1287/trsc.26.3.201.

[22] Kuby, M.-Lim, S.: The Flow-Refueling Location Problem for Alternative-Fuel Vehicles. Socio-Economic Planning Sciences, Vol. 39, 2005, No. 2, pp. 125-145, doi: 10.1016/j.seps.2004.03.001.

[23] Kuby, M.-Lim, S.: Location of Alternative-Fuel Stations Using the Flow-Refueling Location Model and Dispersion of Candidate Sites on Arcs. Networks and Spatial Economics, Vol. 7, 2007, No. 2, pp. 129-152, doi: 10.1007/s11067-006-9003-6. 
[24] Upchurch, C.-Kuby, M.-Lim, S.: A Model for Location of Capacitated Alternative-Fuel Stations. Geographical Analysis, Vol. 41, 2009, No. 1, pp. 85-106, doi: 10.1111/j.1538-4632.2009.00744.x.

[25] Lim, S.-Kuby, M.: Heuristic Algorithms for Siting Alternative-Fuel Stations Using the Flow-Refueling Location Model. European Journal of Operational Research, Vol. 204, 2010, No 1. pp. 51-61, doi: 10.1016/j.ejor.2009.09.032.

[26] Capar, I.-Kuby, M.: An Efficient Formulation of the Flow Refueling Location Model for Alternative-Fuel Stations. IIE Transactions, Vol. 44, 2012, No. 8, pp. 622-636, doi: 10.1080/0740817X.2011.635175.

[27] Mak, H. Y.-Rong, Y.-Shen, Z. J. M.: Infrastructure Planning for Electric Vehicles with Battery Swapping. Management Science, Vol. 59, 2013, No. 7, pp. 1557-1575, doi: $10.1287 /$ mnsc. 1120.1672

[28] Tu, W.- Li, Q.-Fang, Z.-Shaw, S.-Zhou, B.-Chang, X.: Optimizing the Locations of Electric Taxi Charging Stations: A Spatial-Temporal Demand Coverage Approach. Transportation Research Part C: Emerging Technologies, Vol. 65, 2016, pp. 172-189, doi: 10.1016/j.trc.2015.10.004

[29] Shahraki, N.-Cai, H.-Turkay, M.-Xu, M.: Optimal Locations of Electric Public Charging Stations Using Real World Vehicle Travel Patterns. Transportation Research Part D: Transport and Environment, Vol. 41, 2015, pp. 165-176, doi: 10.1016/j.trd.2015.09.011.

[30] Cai, H.-Jia, X.-Chiu, A. S. F.-Hu, X.-Xu, M.: Siting Public Electric Vehicle Charging Stations in Beijing Using Big-Data Informed Travel Patterns of the Taxi Fleet. Transportation Research Part D: Transport and Environment, Vol. 33, 2014, pp. 39-46, doi: 10.1016/j.trd.2014.09.003.

[31] Yang, J.-Dong, J.-Hu, L.: A Data-Driven Optimization-Based Approach for Siting and Sizing of Electric Taxi Charging Stations. Transportation Research Part C: Emerging Technologies, Vol. 77, 2017, pp. 462-477, doi: 10.1016/j.trc.2017.02.014

[32] Dong, J.-Liu, C.-Lin, Z.: Charging Infrastructure Planning for Promoting Battery Electric Vehicles: An Activity-Based Approach Using Multiday Travel Data. Transportation Research Part C: Emerging Technologies, Vol. 38, 2014, pp. 44-55, doi: $10.1016 /$ j.trc.2013.11.001

[33] Xu, M.-Meng, Q.-Liu, K.-Yamamoto, T.: Joint Charging Mode and Location Choice Model for Battery Electric Vehicle Users. Transportation Research Part B: Methodological, Vol. 103, 2017, pp. 68-86, doi: 10.1016/j.trb.2017.03.004.

[34] Мотолкі, Y.: Location-Allocation of Electric Vehicle Fast Chargers - Research and Practice. World Electric Vehicle Journal, Vol. 10, 2019, No. 1, Art. No. 12, doi: 10.3390/wevj10010012.

[35] He, J.-Yang, H.-Tang, T. Q.-Huang, H. J.: An Optimal Charging Station Location Model with the Consideration of Electric Vehicle's Driving Range. Transportation Research Part C: Emerging Technologies, Vol. 86, 2018, pp. 641-654, doi: 10.1016/j.trc.2017.11.026.

[36] He, F.-Wu, D.-Yin, Y.-Guan, Y.: Optimal Deployment of Public Charging Stations for Plug-In Hybrid Electric Vehicles. Transportation Research Part B: Methodological, Vol. 47, 2013, pp. 87-101, doi: 10.1016/j.trb.2012.09.007. 
[37] He, F.-Yin, Y.-Lawphongpanich, S.: Network Equilibrium Models with Battery Electric Vehicles. Transportation Research Part B: Methodological, Vol. 67, 2014, pp. 306-319, doi: 10.1016/j.trb.2014.05.010.

[38] He, F.-Yin, Y.-Zhou, J.: Deploying Public Charging Stations for Electric Vehicles on Urban Road Networks. Transportation Research Part C: Emerging Technologies, Vol. 60, 2015, pp. 227-240, doi: 10.1016/j.trc.2015.08.018.

[39] Fredriksson, H.-Dahl, M.-Holmgren J.: Optimal Placement of Charging Stations for Electric Vehicles in Large-Scale Transportation Networks. Procedia Computer Science, Vol. 160, 2019, pp. 77-84, doi: 10.1016/j.procs.2019.09.446.

[40] López Millán, V.M.-Cholvi, V.-López, L.-Fernández Anta, A.: A Model of Self-Avoiding Random Walks for Searching Complex Networks. Networks, Vol. 60, 2012, No. 2, pp. 71-85, doi: 10.1002/net.20461.

[41] MATLAB, Version 9.4.0813654 (R2018a). The MathWorks Inc., Natick, Massachusetts, 2018.

[42] Inc. Gurobi Optimization. Gurobi Optimizer Reference Manual, 2018. Available at: http://www.gurobi.com. 

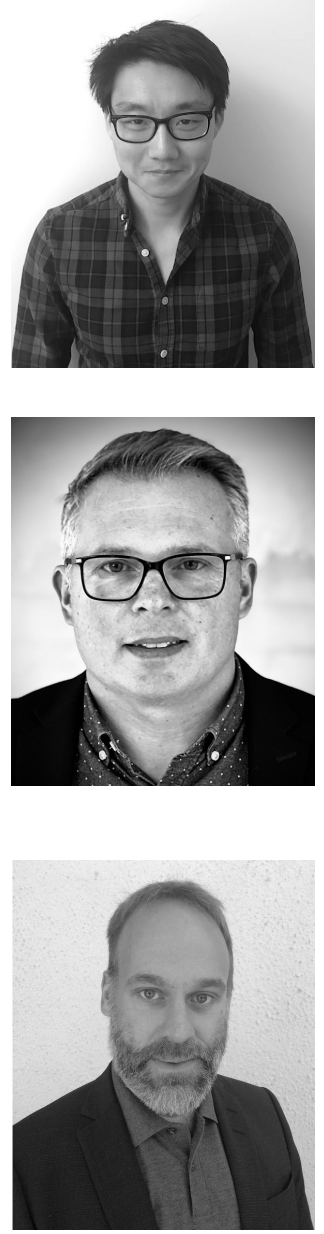

Henrik Fredriksson received his B.Sc. in media technology from Blekinge Institute of Technology in 2011, and B.Sc. and M.Sc. in mathematics from Linnaeus University in 2012 and 2013, respectively. He is currently Ph.D. student in applied mathematics at the Department of Mathematics and Natural Sciences, Blekinge Institute of Technology. His scientific research is focused on mathematical models within the areas of transportation and traffic.

Mattias DAHL received his M.Sc. in computer engineering from Luleå Institute of Technology, 1993, Licentiate in Engineering, Lund University, 1997, and Ph.D. in applied signal processing, Blekinge Institute of Technology (BTH), 2000. Since 2005, he has been with the Department of Mathematics and Natural Sciences, BTH, where he is currently Professor of Systems Engineering. He has authored about 100 scientific publications and patents and has received several awards from the Swedish Innovation Agency and the Swedish Foundation of Technology Transfer.

Johan Holmgren is Associate Professor of computer science at Malmö University, Sweden. He received his Ph.D. degree in computer science at Blekinge Institute of Technology in 2010. Currently, he is employed at the Department of Computer Science and Media Technology at Malmö University. His research interests include agent-based simulation and modeling, mathematical optimization, simulation, and machine learning, and his application areas include freight transport and traffic modeling, public transport, and health-care logistics. 